\title{
Monte Carlo analysis of a new model-based method for insulin sensitivity testing
}

\author{
Thomas F. Lotz ${ }^{a, *}$, J.Geoffrey Chase ${ }^{a}$, Kirsten A. McAuley ${ }^{b}$, Geoffrey M. Shaw ${ }^{c}$, \\ Xing-Wei Wong ${ }^{a}$, Jessica Lin ${ }^{a}$, Aaron LeCompte ${ }^{a}$, Christopher E. Hann ${ }^{a}$, Jim I. Mann ${ }^{b}$ \\ ${ }^{a}$ Centre for Bioengineering, Department of Mechanical Engineering, University of Canterbury, Christchurch, New Zealand \\ b Edgar National Centre for Diabetes Research, University of Otago, Dunedin, New Zealand \\ c Christchurch School of Medicine and Health Science, University of Otago, Christchurch, New Zealand
}

\begin{abstract}
Article history:
Received 17 July 2006

Received in revised form

29 December 2006

Accepted 12 March 2007

Keywords:

Insulin sensitivity

Insulin resistance

Diabetes screening

Glucose modeling

Insulin modeling
\end{abstract}

A R T I C L E I N F O

\begin{abstract}
A B S T R A C T
Insulin resistance (IR), or low insulin sensitivity, is a major risk factor in the pathogenesis of type 2 diabetes and cardiovascular disease. A simple, high resolution assessment of IR would enable earlier diagnosis and more accurate monitoring of intervention effects. Current assessments are either too intensive for clinical settings (Euglycaemic Clamp, IVGTT) or have too low resolution (HOMA, fasting glucose/insulin). Based on high correlation of a model-based measure of insulin sensitivity and the clamp, a novel, clinically useful test protocol is designed with: physiological dosing, short duration $(<1 \mathrm{~h})$, simple protocol, low cost and high repeatability. Accuracy and repeatability are assessed with Monte Carlo analysis on a virtual clamp cohort $(N=146)$. Insulin sensitivity as measured by this test has a coefficient of variation (CV) of $\mathrm{CV}_{\mathrm{SI}}=4.5 \%$ (90\% CI: 3.8-5.7\%), slightly higher than clamp ISI $\left(\mathrm{CV}_{\mathrm{ISI}}=3.3 \%\right.$ (90\% CI: $\left.\left.3.0-4.0 \%\right)\right)$ and significantly lower than HOMA $\left(\mathrm{CV}_{\mathrm{HOMA}}=10.0 \%(90 \%\right.$ CI: $9.1-10.8 \%)$ ). Correlation to glucose and unit normalised ISI is $r=0.98$ (90\% CI: 0.97-0.98). The proposed protocol is simple, cost effective, repeatable and highly correlated to the gold-standard clamp.
\end{abstract}

@ 2007 Elsevier Ireland Ltd. All rights reserved.

\section{Introduction}

\begin{abstract}
A major risk factor in the pathogenesis of type 2 diabetes and cardiovascular disease is insulin resistance (IR) [1]. IR, or low insulin sensitivity, is defined as a reduced ability of the cells to utilise insulin to take up glucose as energy. The pancreas tries to compensate for increasing IR by increasing its insulin production, often leading to an exhaustion of the insulin producing beta-cells, resulting in impaired fasting glucose (IFG). An early diagnosis of IR can enable early intervention and delay the onset of diabetes, thus greatly reducing the effects and cost of further complications.
\end{abstract}

The ability to sensitively and accurately identify individuals with IR is critical for the implementation and assessment of intervention programmes in high risk groups. To diagnose IR in population studies and to be applicable in clinical settings, a test has to be simple and cost effective. However, it must also be accurate enough to assess small changes in IR or the progression of treatment.

The euglycaemic-hyperinsulinaemic clamp (clamp) [2] is the gold-standard to assess insulin sensitivity. However, it is clinically very intense and thus not practicable for large populations $[3,4]$ or typical clinical use. Hence, simpler methods have emerged, such as the insulin tolerance test (ITT)

\footnotetext{
* Corresponding author. Tel.: +64 3364 2987x7486.

E-mail address: tlo16@student.canterbury.ac.nz (T.F. Lotz). 0169-2607/\$ - see front matter (C) 2007 Elsevier Ireland Ltd. All rights reserved.

doi:10.1016/j.cmpb.2007.03.007
} 
[5], the intravenous glucose tolerance test (IVGTT) [6-8] and the oral glucose tolerance test (OGTT) [9-11]. The intravenous tests mostly use the minimal model of glucose kinetics to quantify insulin resistance [12], whereas the OGTT has been analysed with a variety of approaches $[10,13]$. All these tests rely on a steady state glucose concentration in the end to avoid unmodeled feedback dynamics in insulin and glucose after a perturbation, either through insulin and glucose infusions, bolus injections or oral glucose loads. They require $2-4 \mathrm{~h}$ to perform. The model-based methods require frequent sampling of glucose and insulin.

Simple, fasting assessments include Homeostasis Model Assessment (HOMA) [14] and Quantitative Insulin Sensitivity Check Index (QUICKI) [15]. These metrics require only one fasting sample of glucose and insulin, but are less accurate and repeatable due to varying fasting conditions, i.e. a pulsatile insulin secretion pattern [16], natural variability in fasting glucose or simply assay errors $[17,18]$. To be a useful clinical tool for repeated and easy use, these measures must correlate well with the gold-standard clamp test. To date, they have not achieved enough resolution to accurately assess a wide range in insulin sensitivity, limiting their application to a rough initial estimation of at-risk populations.

However, they are the primary avenue for diagnosis of type 2 diabetes and IFG. As a result of their low resolution the diagnosis can occur very late in the aetiology of the disease, and after some physiological damage has occurred $[19,20]$. Combined with incomplete compliance with regular medical checks, many individuals in at-risk groups can go undiagnosed for several years [21,20].

This research engineers a new model-based insulin sensitivity test, relying on the dynamic state after a low-dose glucose and insulin input. During this transient state, the metabolic system model employed has been shown to be highly correlated $(r=0.97)$ to the clamp test $[22,23]$. The goal is a highly accurate, short and cost effective test that is useful in a clinical setting and overcomes the limitations of currently used metrics.

The main performance criteria for this test are thus accuracy and repeatability. To assess these criteria, a Monte Carlo analysis is performed on test simulations, taking into account significant errors. These errors include: errors in laboratory assays, dilution of input solutions, timing of samples, and errors due to unmodeled dynamics. These latter errors could include poorly or unmodeled endogenous glucose production (EGP) and first pass hepatic insulin extraction. The simulations are based on model-based insulin sensitivity values obtained from fitting a cohort of 146 euglycaemic-hyperinsulinaemic clamp tests, covering a range of metabolic responses. Rather than assessing clinical/physiological validity of the estimated insulin sensitivity, this study aims at validating the robustness of the proposed test in a noisy clinical test environment.

\section{Methodology}

\subsection{Procedure}

The simulation procedure used in this study is shown in the schematic in Fig. 1, and explained in more detail in the follow-

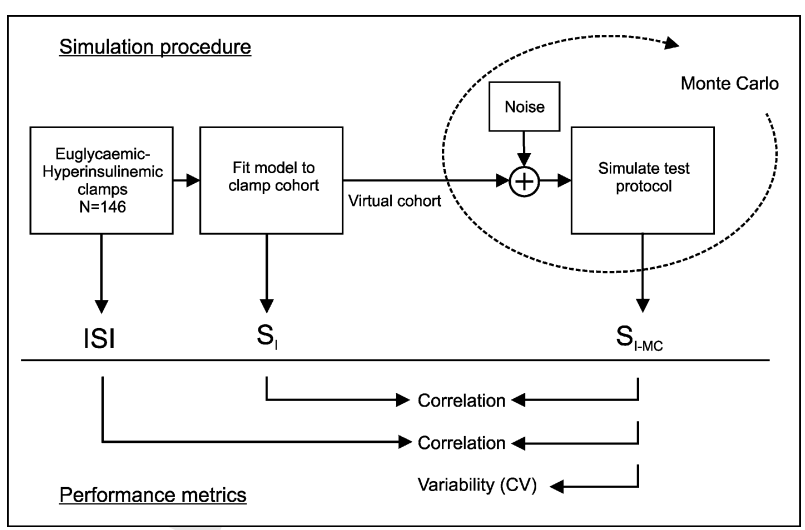

Fig. 1 - Simulation procedure and performance metrics used in this study.

ing sections:

(1) Insulin sensitivity from 146 euglycaemic-hyperinsulinaemic clamp tests [24] is calculated from the test data (ISI).

(2) A virtual cohort is created by fitting the metabolic model to the clamp tests, resulting in a model-based insulin sensitivity for each subject $\left(\mathrm{S}_{\mathrm{I}}\right)$.

(3) Monte Carlo simulations on the proposed test protocol are run on the virtual cohort by adding random noise from published error levels to measurements and inputs, and accounting for potentially unmodeled regulatory dynamics.

(4) The metabolic model is fit to the simulated test profiles (glucose, insulin and C-peptide concentrations), resulting in insulin sensitivity from the proposed low intensity test ( $\mathrm{S}_{\mathrm{I}-\mathrm{MC}}$ ).

(5) Performance of the method is assessed by the coefficient of variation (CV = S.D. $/$ mean) of $\mathrm{S}_{\mathrm{I}-\mathrm{MC}}$ and by correlating $\mathrm{S}_{\mathrm{I}-\mathrm{MC}}$ with ISI and $\mathrm{S}_{\mathrm{I}}$. Additional comparisons are made to HOMA.

\subsection{Test protocol}

The test is designed to be a short dynamic test to assess insulin sensitivity from a metabolic system model fit to the transient plasma glucose and insulin curves after intravenous (IV) bolus injections of glucose and insulin. The model then relates interstitial insulin to plasma glucose to determine the subject's sensitivity to insulin. The protocol has to account for a wide variety of individuals (lean, obese, insulin resistant, diabetic) and be short, robust and simple enough to be applicable in a clinical setting. The dosing should be lower than in an IVGTT to assess a more physiological state and to minimise regulatory responses, such as suppression of endogenous glucose production (EGP) and pancreatic insulin secretion.

The protocol used in this development study results in metabolic dose-response curves as shown for glucose and insulin in the example in Fig. 2. It is 55 min long and includes:

(1) Inject a fixed dose of glucose $(5,10$ or $20 \mathrm{~g})$ at $0 \mathrm{~min}$.

(2) Inject a fixed dose of insulin $(0.5,1$ or $2 \mathrm{U})$ at $10 \mathrm{~min}$. 

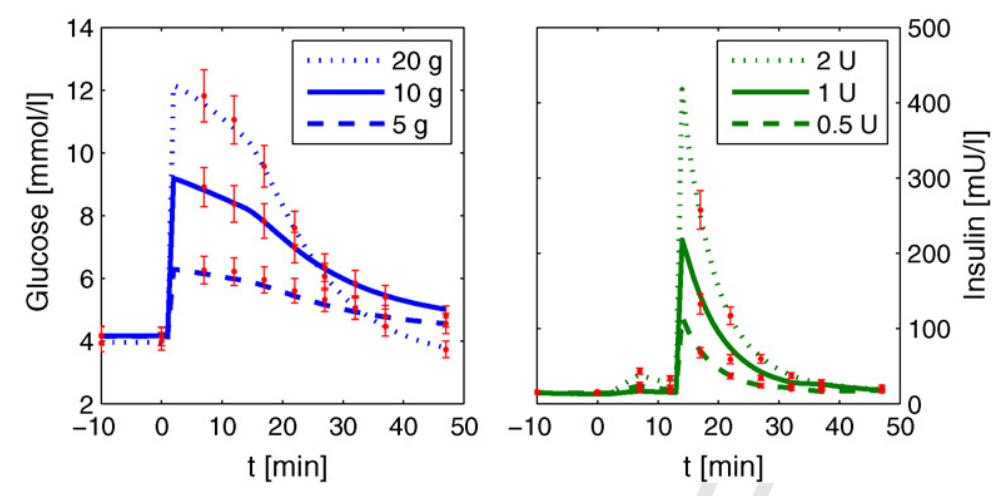

Fig. 2 - Example of simulated profiles of glucose (left) and insulin (right) responses to the low-, medium- and high-dose test protocols on the same virtual subject. Discrete measurements are shown with error bars along with model fits (continuous curves).

(3) Sample blood at -10, 0, 5, 10, 15, 20, 25, 30, 35, $45 \mathrm{~min}$ and assay for glucose, insulin and C-peptide concentrations.

(4) Fit metabolic models of glucose, insulin and C-peptide to dose-response curves.

(5) Determine insulin sensitivity from model parameter $S_{I}$.

A more frequent sampling directly after the injections, as done in an IVGTT is not practicable, as the mixing process in plasma can take up to 5-8 min to complete, and earlier measurements may thus be inaccurate [25].

The dosing of $10 \mathrm{~g}$ of glucose and $1 \mathrm{U}$ of insulin was chosen as it is physiological and minimises the risk of hypoglycaemia. It is also large enough to provide a good signal to noise ratio. The protocol was also simulated with half $(5 \mathrm{~g}$ glucose $/ 0.5 \mathrm{U}$ insulin) and twice (20 g glucose/2 $\mathrm{U}$ insulin) the dose to assess differences in expected accuracy. The 55-min length and sample numbers are primarily to maximise data to engineer a final 30-45 min, less intense test with fewer samples.

\subsection{System model}

A simpler form of the glucose-insulin metabolic system model shown in Fig. 3 has been presented previously and validated on a wide range of subjects, namely on retrospective intensive

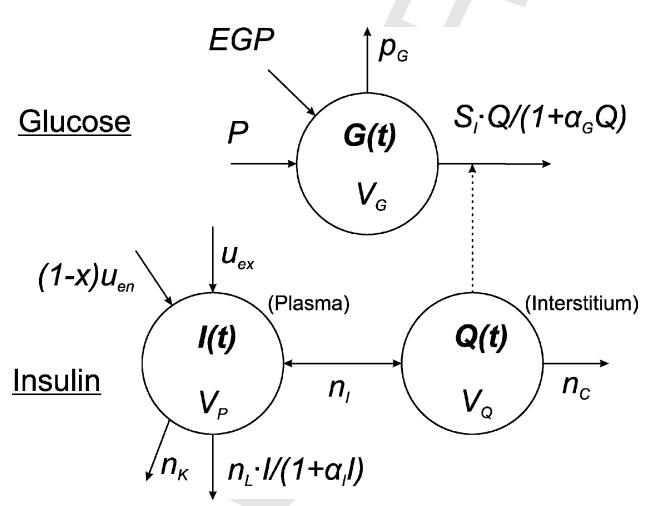

Fig. 3 - Schematic of the glucose-insulin model employed in this study. The three compartments represent different volumes of distribution. Parameters are explained in detail in Section 2.3. care unit (ICU) data [26], in glycaemic control trials in critical care [27-30] and on euglycaemic-hyperinsulinaemic clamp and IVGTT data on healthy, insulin resistant and type 2 diabetes subjects [22,31]. To account for metabolic differences between critically ill and healthy subjects, a time-varying insulin sensitivity parameter was employed in validations on critically ill subjects. On non-ICU populations, this correction was not necessary and the model was able to accurately account for all dynamics $[22,31]$.

The glucose-insulin pharmacodynamic model is derived from the Minimal Model by Bergman et al. [6]. This model is further enhanced by glucose clearance saturation dynamics in the form of a Michaelis-Menten equation [32,33].

The two compartment insulin pharmacokinetics model used here is the primary enhancement from the original system model. It is derived from earlier studies by Sherwin et al. [34]. The accessible central compartment can be understood as plasma plus fast exchanging tissues. The peripheral compartment represents interstitial fluid. The model accounts for the major losses of insulin from the central compartment by the liver and the kidneys and the loss out of the peripheral compartment, mainly insulin binding and eventual degradation by the cells. Transport between the compartments is assumed to be bi-directional diffusion. The resulting system model is defined by the following equations [31]:

$$
\begin{aligned}
& \dot{G}=-p_{G} G-S_{I}\left(G+G_{E}\right) \frac{Q}{1+\alpha_{G} Q}+\frac{P}{V_{G}}+E G P, \quad G(0)=0 \\
& \dot{Q}=-n_{C} Q+\frac{n_{I}}{V_{Q}}(I-Q), \quad Q(0)=\frac{3}{5} I_{E} \\
& \dot{I}=-n_{K} I-\frac{n_{L} I}{1+\alpha_{I} I}-\frac{n_{I}}{V_{P}}(I-Q)+\frac{u_{e x}}{V_{P}}+(1-x) \frac{u_{e n}}{V_{P}}, \quad I(0)=I_{E}
\end{aligned}
$$

where $G$ is the concentration of plasma glucose above equilibrium level $G_{\mathrm{E}}\left(\mathrm{mmoll}^{-1}\right)$; I the concentration of plasma insulin $\left(\mathrm{mUl}^{-1}\right) ; \mathrm{Q}$ the concentration of insulin in interstitial fluid $\left(\mathrm{mUl}^{-1}\right) ; \mathrm{G}_{\mathrm{E}}$ the equilibrium (fasting) plasma glucose concentration $\left(\mathrm{mmoll}^{-1}\right) ; \mathrm{I}_{\mathrm{E}}$ the equilibrium (fasting) plasma insulin concentration $\left(\mathrm{mU}^{-1}\right)$; $u_{\mathrm{ex}}, u_{\mathrm{en}}$ the exogenous, endogenous 
insulin input rate $\left(\mathrm{mU} \mathrm{min}^{-1}\right) ; \mathrm{P}$ the exogenous glucose input rate $\left(\mathrm{mmol} \mathrm{min}^{-1}\right)$; EGP the endogenous glucose production rate $\left(\mathrm{mmoll}^{-1} \mathrm{~min}^{-1}\right) ; p_{\mathrm{G}}$ the clearance rate of plasma glucose at basal insulin $\left(\mathrm{min}^{-1}\right) ; S_{I}$ the insulin sensitivity $\left(1 \mathrm{mU}^{-1} \mathrm{~min}^{-1}\right) ; V_{\mathrm{P}}$ the plasma volume (+Fast exchanging tissues) (1); $V_{Q}$ the interstitial fluid volume (1); $V_{G}$ the glucose distribution volume (l); $x$ the fractional first pass hepatic insulin extraction (decimal \% value); $n_{\mathrm{K}}$ the kidney clearance rate of insulin from plasma $\left(\mathrm{min}^{-1}\right) ; n_{\mathrm{L}}$ the liver clearance rate of insulin from plasma $\left(\mathrm{min}^{-1}\right) ; n_{\mathrm{I}}$ the diffusion constant of insulin between compartments $\left(\mathrm{min}^{-1}\right)$; $n_{\mathrm{C}}$ the cellular insulin clearance rate from interstitium $\left(\mathrm{min}^{-1}\right) ; \alpha_{\mathrm{I}}$ the Michaelis-Menten parameter for liver clearance rate saturation $\left(1 \mathrm{mU}^{-1}\right) ; \alpha_{\mathrm{G}}$ is the Michaelis-Menten parameter for insulin-stimulated glucose clearance saturation $\left(\mathrm{mU}^{-1}\right)$.

\subsection{Parameter fitting and identification}

The parameters are identified a priori where possible and using an integral based fitting method for patient specific parameters, as described in refs. [26,22]. In the insulin model parameters $\mathrm{V}_{\mathrm{P}}, \mathrm{V}_{\mathrm{Q}}, n_{\mathrm{I}}, n_{\mathrm{K}}$ are assumed to be identical to corresponding values for C-peptide, due to the similar molecular weight of insulin (5800 Da [35]) and C-peptide (3600 Da [35]) and their similar passive properties. The parameters are taken from a well validated population model of C-peptide kinetics [36]. Variable $n_{C}$ is calculated to achieve a steady state concentration ratio of $\mathrm{I} / \mathrm{Q}=5 / 3$ [37-39], and $\alpha_{\mathrm{I}}=0.0017$ is a mean population value from $[40,26]$

In the glucose model, parameter $p_{G}=0.01$ is fixed to an approximate population value $[40,26]$. Note that $p_{\mathrm{G}}$ can be estimated on some data sets, as in clinical glycaemic control trials [41,42], but the data in this study does not have the resolution to uniquely identify it. In addition, it is not a dominant dynamic in the presence of low doses and exogenous insulin [32,33]. Equilibrium glucose concentration $G_{E}$ is set to the fasting glucose level of each subject, as shown in the cohort description in Table 1. Glucose clearance saturation is set to $\alpha_{\mathrm{G}}=0$ in this study, as the subjects are fasted and with the low dose injection, saturation is not very likely. This value also better matches the assumptions used in calculating ISI for the supra-physiological clamp test [2].

The remaining parameters, $n_{\mathrm{L}}$ and $x$ for insulin and $S_{\mathrm{I}}$ and $V_{G}$ for glucose are identified using the integral based fitting method described in more detail by Hann et al. [26].
Briefly, the differential equations are integrated in different time-steps by interpolating between discrete measurements, transforming the non-convex non-linear problem into a set of linear equations that can be easily solved using linear least squares (LS). The method is convex and not starting point dependent, unlike commonly used non-linear recursive least squares (NRLS). Errors in the integration of equations, i.e. due to noise, are not critical, as the method minimises areas under the curve, not absolute differences, thus effectively filtering noisy data $[26,43]$. The errors have been shown to be, in the limit, on the order of model error [26]. Integrating Eq. (1) in the interval $\left[t_{0}, t_{1}\right]$ yields:

$$
\begin{aligned}
G\left(t_{1}\right)-G\left(t_{0}\right)= & -p_{G} \int_{t_{0}}^{t_{1}} G(t) d t-S_{I} \int_{t_{0}}^{t_{1}}\left(G(t)+G_{E}\right) \frac{Q(t)}{1+\alpha_{G} Q(t)} d t \\
& +\frac{1}{V_{G}} \int_{t_{0}}^{t_{1}} P(t) d t+\int_{t_{0}}^{t_{1}} E G P(t) d t
\end{aligned}
$$

This step can be repeated for different time intervals, resulting in a set of linear equations that can be readily solved.

$\overline{\mathrm{A}}\left[\mathrm{S}, \frac{1}{\mathrm{~V}_{\mathrm{G}}}\right]^{\mathrm{T}}=\overline{\mathrm{b}}$

The same method is applied to the measured plasma insulin profile $I(t)$ to estimate parameters $n_{\mathrm{L}}$ and $x$, using the analytical solution for $Q(t)$ in integrating Eq. (3).

$Q(t)=\frac{n_{I}}{V_{Q}} \int_{0}^{t} I(\tau) e^{-\left(n_{C}+\left(n_{I} / V_{Q}\right)\right)(t-\tau)} d \tau$

234 235 236

The result is a set of linear equations:

$\bar{B}\left[n_{L}, x\right]^{T}=\bar{c}$

The time intervals used in the integrations can be chosen to suit the available data density, as long as the minimum number of intervals required are used to ensure an optimal LS solution [26]. In this study, two sets of measured data are available $(I(t)$ and $G(t))$ to estimate two parameters in each. The optimal interval length was identified as $2 \mathrm{~min}$ in this study, resulting in 28 integral equation for each data set. No additional weighting or normalisation was performed with the equations.

Table 1 - Description of the intervention study population from McAuley et al. [24], used to create the virtual simulation cohort in this study

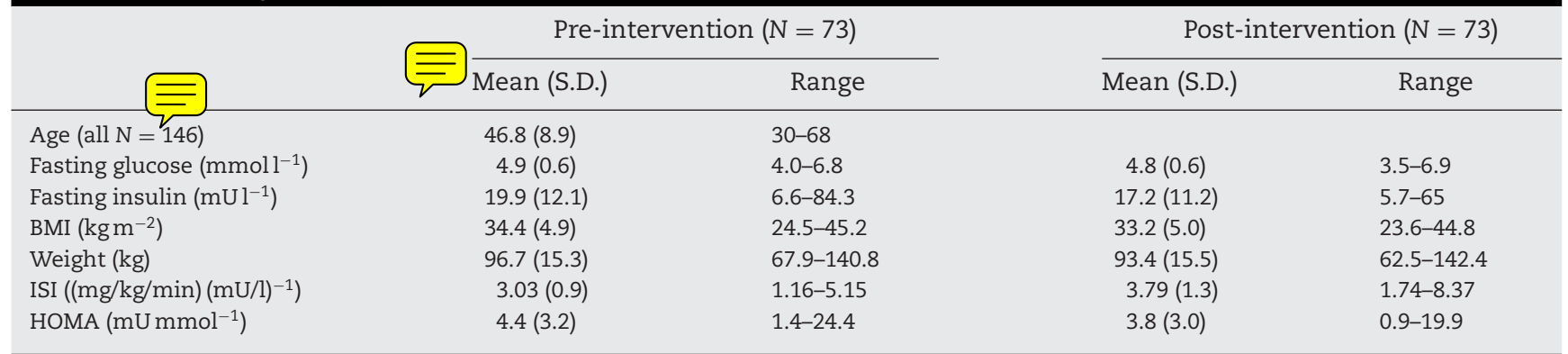




\subsection{Test simulation on clamp cohort}

To simulate the proposed test and make it comparable to the clamp, a simulation cohort was created using metabolic information estimated from a set of clamp trials performed by McAuley et al. [24] to study the effects of lifestyle interventions on insulin resistance. The data consist of 146 trials performed on 73 individuals, once before and once after a 16 week intervention. Details of the clamp study population are given in Table 1.

The clamp is a test to determine insulin sensitivity in a research setting. It is currently the most accurate method to measure insulin sensitivity, but too intense and expensive to be used in a clinical setting. A constant insulin infusion is paired with a varying glucose infusion to achieve a steady state glucose concentration. Blood is sampled every $10 \mathrm{~min}$ for 2-4 h. Due to the supra-physiological dosing, it is assumed that endogenous glucose and insulin are fully suppressed after $60 \mathrm{~min}$. The insulin sensitivity index (ISI) derived from this test is the ratio of the average glucose infusion rate to the mean plasma insulin concentration during steady state $\left(\mathrm{ISI}=\mathrm{P}_{\mathrm{SS}} / \mathrm{I}_{\mathrm{SS}}\right)$.

The clamp trials were fitted by the model described in Eqs. (1)-(3) by estimating parameters $S_{I}$ and $n_{L}$. Mean absolute errors of the fits were 5.9\% (S.D. 6.6\%) for glucose and 6.2\% (S.D. $6.4 \%)$ for insulin [22]. Insulin sensitivity, $S_{I}$, was estimated as time-varying, piecewise constant during transient and steady state [31]. The steady state value was taken for the subsequent simulations. Mean $n_{\mathrm{L}}$ estimated from the clamps was very low compared to that seen on dose-response tests. This can be caused by various factors, i.e. heavy saturation of the liver being exposed to such large supra-physiological concentrations [44], reduced clearance in obesity [45] or incomplete suppression of pancreatic insulin during the clamp, which is not accounted for in the model fit and results in underestimated $n_{\mathrm{L}}$. Incomplete suppression of pancreatic insulin secretion is particularly likely, given the shorter and lower dose method used in this clamp study [3].

To achieve a more realistic insulin profile, $n_{\mathrm{L}}$ was thus increased by $0.1 \mathrm{~min}^{-1}$ for all individuals based on empirical testing on various dose-response data (unpublished). This increase results in a more realistic simulated insulin profile and does not change the insulin sensitivity. Thus, the outcome of the test and the performance of the simulations are not affected. Due to the low resolution of the clamp data, further parameters had to be identified a priori, as described in Section 2.4. In addition, $V_{G}$ was set to $V_{G}=1.2\left(V_{P}+V_{Q}\right)$, as the clamp data is not dense enough to allow a unique identification. A $20 \%$ larger volume than the total insulin distribution volume was chosen, as glucose distribution volume has been found to be larger than for insulin, due to fast hepatic storage and non-insulin dependent uptake by the brain $[46,47]$. This choice does not affect the outcome of the study, as $S_{I}$ and $V_{G}$ are subsequently identified from the test profiles as described in Section 2.4.

Model simulation parameters determined from the clamp population as described in Sections 2.4 and 2.5 are given in Table 2. These parameter values are used to create the virtual cohort on which the test protocol is simulated.

Pancreatic insulin secretion is not known for this cohort, as C-peptide data is not available. A healthy pancreas responds to a glucose input by secreting insulin in two phases. The first phase consists of an insulin burst, lasting approximately $10 \mathrm{~min}$, followed by a second phase of lower dose, but longer duration [35]. Insulin secretion can be suppressed or reduced by exogenous insulin, with a full suppression only achievable by a prolonged infusion of large amounts of insulin [3]. In the protocol for this study, an insulin bolus is injected $10 \mathrm{~min}$ after glucose, thus not affecting first phase burst, but suppressing second phase insulin secretion. Simulated total insulin secretion rate is thus reduced back to its basal rate after the bolus injection of exogenous insulin $[23,48,49]$.

Pre-hepatic endogenous insulin secretion can be simulated by a basal secretion rate, superimposed by a first-phase burst. The burst peaks at a rate of $72 \mathrm{mU} \mathrm{min}^{-1} \mathrm{~m}^{-2} \mathrm{BSA}[50,51]$, which is dependent on body surface area (BSA), and is followed by an exponential decay lasting $10 \mathrm{~min}$. For the lower and higher dose protocol, this first-phase burst is halved and doubled, respectively [52]. Basal endogenous secretion $u_{\mathrm{b}}$ is calculated from the steady state fasting insulin balance using Eq. (3) with insulin concentrations $I_{b}$ and $Q_{b}=(3 / 5) I_{b}$, and $a$

Table 2 - Simulation model parameters calculated and estimated as described in Sections 2.4 and 2.5 to generate the virtual simulation cohort

\begin{tabular}{|c|c|c|c|c|}
\hline & \multicolumn{2}{|c|}{ pre-intervention $(\mathrm{N}=73)$} & \multicolumn{2}{|c|}{ post-intervention $(\mathrm{N}=73)$} \\
\hline & Mean (S.D.) & Range & Mean (S.D.) & Range \\
\hline $\mathrm{V}_{\mathrm{P}}(\mathrm{l})$ & $4.52(0.37)$ & $3.98-5.93$ & $4.46(0.36)$ & $3.90-5.96$ \\
\hline $\mathrm{V}_{\mathrm{Q}}(\mathrm{l})$ & $5.67(0.54)$ & $4.52-7.47$ & $5.54(0.57)$ & $4.44-7.26$ \\
\hline $\mathrm{V}_{\mathrm{G}}(\mathrm{l})$ & $12.22(1.06)$ & $10.20-15.67$ & $12.00(1.08)$ & $10.00-15.75$ \\
\hline$n_{I}\left(\operatorname{lmin}^{-1}\right)$ & $0.28(0.027)$ & $0.22-0.36$ & $0.27(0.029)$ & $0.21-0.36$ \\
\hline$n_{K}\left(\min ^{-1}\right)$ & $0.060(0.0024)$ & $0.053-0.064$ & $0.060(0.0028)$ & $0.053-0.064$ \\
\hline$n_{\mathrm{L}}\left(\min ^{-1}\right)$ & $0.15(0.027)$ & $0.10-0.21$ & $0.16(0.022)$ & $0.10-0.20$ \\
\hline$n_{C}\left(\min ^{-1}\right)$ & $0.032(0.00037)$ & $0.032-0.033$ & $0.032(0.00038)$ & $0.032-0.033$ \\
\hline $\mathrm{S}_{\mathrm{I}}\left(10^{-4} 1 \mathrm{mU}^{-1} \mathrm{~min}^{-1}\right)$ & $4.91(1.54)$ & $2.08-8.29$ & $6.18(2.13)$ & $3.07-13.0$ \\
\hline$p_{\mathrm{G}}\left(\min ^{-1}\right)$ & & & & \\
\hline$\alpha_{\mathrm{I}}\left(\mathrm{lmU}^{-1}\right)$ & & & & \\
\hline$\alpha_{\mathrm{G}}\left(1 \mathrm{mU}^{-1}\right)$ & & & & \\
\hline
\end{tabular}


randomly generated first pass hepatic extraction $x$ :

$u_{\mathrm{b}}=\frac{\mathrm{V}_{\mathrm{P}} \mathrm{I}_{\mathrm{b}}}{1-x}\left(n_{\mathrm{K}}+\frac{n_{\mathrm{L}}}{1+\alpha_{\mathrm{I}} \mathrm{I}_{\mathrm{b}}}+\frac{2}{5} \frac{n_{\mathrm{I}}}{\mathrm{V}_{\mathrm{P}}}\right)$

Total pre-hepatic endogenous insulin secretion is thus modeled as:

$u_{e n}(t)= \begin{cases}u_{b}+(72 B S A) e^{-0.3 t} & 0 \leq t<10 \min \\ u_{b} & t<0 \text { and } t \geq 10 \mathrm{~min}\end{cases}$

First pass hepatic extraction is often approximated around $50 \%$, but is generally higher in fasting state [53-55], often reaching values of over $90 \%$ [54]. As a conservative choice, $x$ is thus determined from a uniform distribution of values between 0.5 and 0.95 (almost complete extraction). Using this model, the total insulin secreted and the peak during the first phase match values reported in the literature [50,51].

Basal endogenous glucose production $\mathrm{EGP}_{\mathrm{b}}$ is calculated from the fasting steady state glucose balance in Eq. (1), where $\mathrm{Q}_{\mathrm{b}}=(3 / 5) \mathrm{I}_{\mathrm{b}}$ and $\mathrm{G}(0)=0$ :

$\mathrm{EGP}_{\mathrm{b}}=\mathrm{S}_{\mathrm{I}} G_{\mathrm{E}} \frac{(3 / 5) \mathrm{I}_{\mathrm{b}}}{1+\alpha_{\mathrm{G}}(3 / 5) I_{\mathrm{b}}}$

\subsection{Monte Carlo analysis}

The Monte Carlo analysis simulates test result accuracy in the presence of assay, timing, insulin and glucose dilution errors, and unmodeled suppression of endogenous glucose production. The assay errors are assumed normally distributed with inter- and intra-batch coefficients of variation $\left(\mathrm{CV}_{\text {inter }}, \mathrm{CV}_{\text {intra }}\right)$ reported by the assay manufacturers. Random intra-batch errors are generated for each sample of a test and added to an inter-batch error, equal for all samples of a given test. As $C V_{\text {intra }}$ is assumed to be included in the reported $C V_{\text {inter }}$, the $\mathrm{CV}$ to be superimposed on $\mathrm{CV}_{\text {intra }}\left(\mathrm{CV}_{\text {add }}\right)$ is calculated:

$\mathrm{CV}_{\text {add }}=\sqrt{\mathrm{CV}_{\text {inter }}^{2}-\mathrm{CV}_{\text {intra }}^{2}}$

Errors in timing of samples are caused by variations in blood sampling procedure and are assumed to be normally distributed between $\pm 30 \mathrm{~s}$ around the sampling time. Due to anticipation of these small complications, the sampling procedure is usually initiated early, thus sometimes resulting in early sampling. Dilution errors can occur when drawing up glucose in a syringe or when diluting insulin, which is typically distributed in highly concentrated form (e.g. $100 \mathrm{U} / \mathrm{ml}$ ). Insulin has also been reported to bind to inner walls of syringes and tubes when being administered, causing a loss of insulin during the dilution process [56]. As these are well known problems and usually taken into account by the investigator and the choice of equipment, the errors are assumed to be normally distributed around the mean.

Suppression of EGP is caused by increases in plasma insulin or glucose [49]. The amount and efficiency of suppression is dependent on the administered dose [57]. With the low dose this test aims at, the suppression is likely not as large as during an IVGTT $(75-100 \%[58,59])$, but cannot be neglected. Since this level of suppression cannot be easily measured, a linear reduction of EGP is assumed from the time of insulin input, reaching a randomly generated maximal suppression $\mathrm{EGP}_{\text {suppr }}$ at the end of the test. It is defined:

$\operatorname{EGP}(t)= \begin{cases}\operatorname{EGP}_{\mathrm{b}}\left(1-\mathrm{EGP}_{\text {suppr }} t / t_{\text {end }}\right) & t_{\text {end }} \geq t>0 \mathrm{~min} \\ \mathrm{EGP}_{\mathrm{b}} & t \leq 0 \mathrm{~min}\end{cases}$

The maximal suppression at the $10 \mathrm{~g} / 1 \mathrm{U}$ dose was chosen randomly from a normal distribution between 25 and $75 \%$. For the lower $(5 \mathrm{~g} / 0.5 \mathrm{U})$ and higher $(20 \mathrm{~g} / 2 \mathrm{U})$ dose variants,

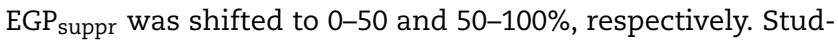
ies have shown a direct dose-dependent relationship between glucose concentration and suppression of EGP [57], validating this basic approach.

The random disturbances thus assumed in this Monte Carlo analysis are:

- Glucose assay errors: $\mathrm{CV}_{\text {intra }}=1 \% ; \mathrm{CV}_{\text {inter }}=2 \%[18]$

- Insulin assay errors: $\mathrm{CV}_{\text {intra }}=2 \% ; \mathrm{CV}_{\text {inter }}=2.8 \%[18,60]$

- C-peptide assay errors: $\mathrm{CV}_{\text {intra }}=3 \% ; \mathrm{CV}_{\text {inter }}=3.4 \%$ [61]

- Glucose input error: $\mathrm{CV}=1.67 \%$

- Insulin input error (dilution): $\mathrm{CV}=3.33 \%$

- Sample timing error: S.D. $10 \mathrm{~s}$

- First pass hepatic insulin extraction: $x \in[0.50,0.95]$

- Maximal suppression of EGP: EGP suppr $=50 \%$ (10 g/1 U); $25 \%$ (5 g/0.5 U); 75\% (20 g/2 U) (S.D. 8.3\%)

The required number of Monte Carlo simulations was identified to be 500 in a convergence test, as the variability in the

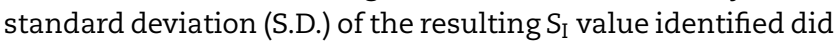
not change significantly with more runs.

An example of the resulting simulated profiles of glucose and insulin responses during the proposed test, employing all three dosing options on one virtual subject, is shown in Fig. 2.

\subsection{Performance metrics and statistics}

Performance of the method was assessed by correlation (Pearson correlation) of the estimated insulin sensitivity $S_{I}$ with the gold-standard clamp test. Accuracy of estimation of $S_{I}$ is given as its coefficient of variation (CV = S.D./mean). The distribution of $S_{I}$ can be assumed to be normal, as assessed by the single sample Kolmogorov-Smirnov (KS) test.

Accuracy of ISI was assessed by Monte Carlo analysis with assay errors as described above and a glucose infusion error of $10 \%$. Accuracy of HOMA is affected by assay errors and pulsatile basal insulin secretion. It is estimated through Monte Carlo analysis with a CV of $10 \%$, as reported by Wallace et al. [17].

\section{Results}

The model parameter for insulin sensitivity fit from clamp trials is $\mathrm{S}_{\mathrm{I}}=5.55$ (S.D.1.95) $\times 10^{-4} \mathrm{lmU}^{-1} \mathrm{~min}^{-1}$. This value is higher than clamp ISI normalised by steady state glucose and corrected for units $\left(\mathrm{ISI}_{\mathrm{G}}=\mathrm{ISI} / \mathrm{G} \times\right.$ weight $\left./ \mathrm{V}_{\mathrm{G}}\right) \mathrm{ISI}_{\mathrm{G}}=$ 3.23(S.D.1.16) $\times 10^{-4} \mathrm{lmU}^{-1} \mathrm{~min}^{-1}$. This difference is due to the different compartmental insulin concentrations used in the respective calculations. The clamp uses plasma insulin (I)
406 


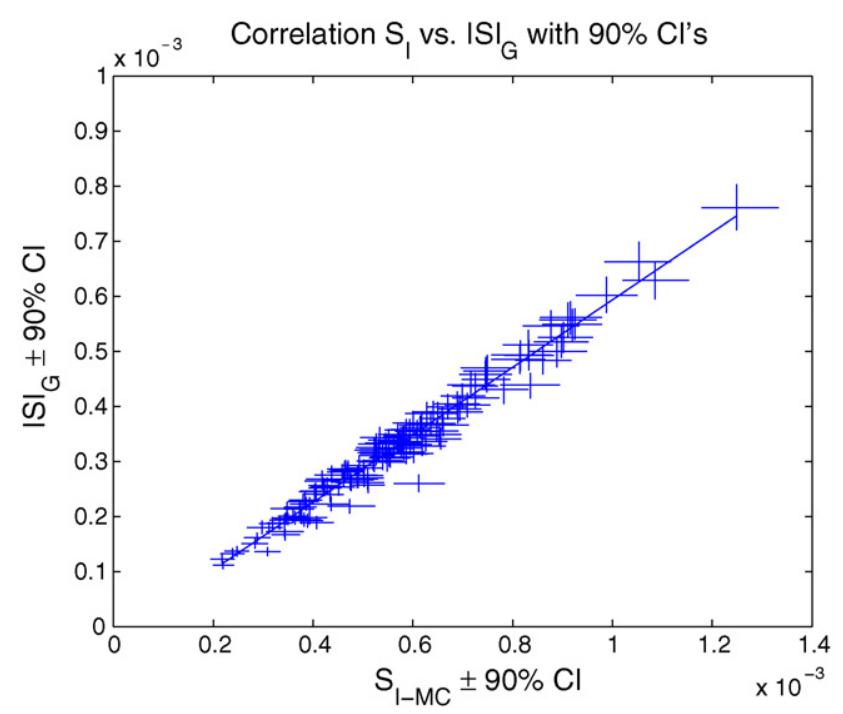

Fig. 4 - Correlation $\mathrm{S}_{\mathrm{I}-\mathrm{MC}}$ and $\mathrm{ISI}_{\mathrm{G}}$ with $90 \% \mathrm{CI}$ 's of each metric and mean regression line.

and the modeled $\mathrm{S}_{\mathrm{I}}$ uses interstitial insulin (Q). Clamp fitted $\mathrm{S}_{\mathrm{I}}$ and measured ISI correlate $r=0.93$. However, $S_{I}$ and ISI $_{G}$ correlate much better $r=0.99$. The higher correlation with $\operatorname{ISI}_{G}$ is a result of the unit correction, which reduces variability introduced by other parameters and imperfect clamping to a basal glucose level [9].

Mean insulin sensitivity resulting from Monte Carlo analysis is $\mathrm{S}_{\mathrm{I}-\mathrm{MC}}=5.56$ (S.D.1.96) $\times 10^{-4} \mathrm{lmU}^{-1} \mathrm{~min}^{-1}$ and thus identical to $\mathrm{S}_{\mathrm{I}}$. Correlations with ISI and $\mathrm{ISI}_{\mathrm{G}}$ are slightly lower, at $r=0.91$ (90\% CI: 0.90-0.92) and $r=0.98$ (90\% CI: 0.97-0.98), respectively. Fig. 4 shows the correlation plot of $\mathrm{S}_{\mathrm{I}-\mathrm{MC}}$ and $\mathrm{ISI}_{\mathrm{G}}$ with the $90 \%$ CI's of each metric.

Intra-individual CV in $\mathrm{S}_{\mathrm{I}-\mathrm{MC}}$ using the proposed low intensity test method is $\mathrm{CV}_{\mathrm{SI}}=4.5 \%$ (90\% CI: $\left.3.8-5.7 \%\right)$. This value is larger than the CV for ISI, CV ISI $=3.3 \%(90 \%$ CI: $3.0-4.0 \%)$, but significantly lower than the CV for HOMA, $\mathrm{CV}_{\text {HOMA }}=10.0 \%$ (90\% CI: 9.1-10.8\%). The three intra-individual CV's are shown in Fig. 5 for all $N=146$ subjects.

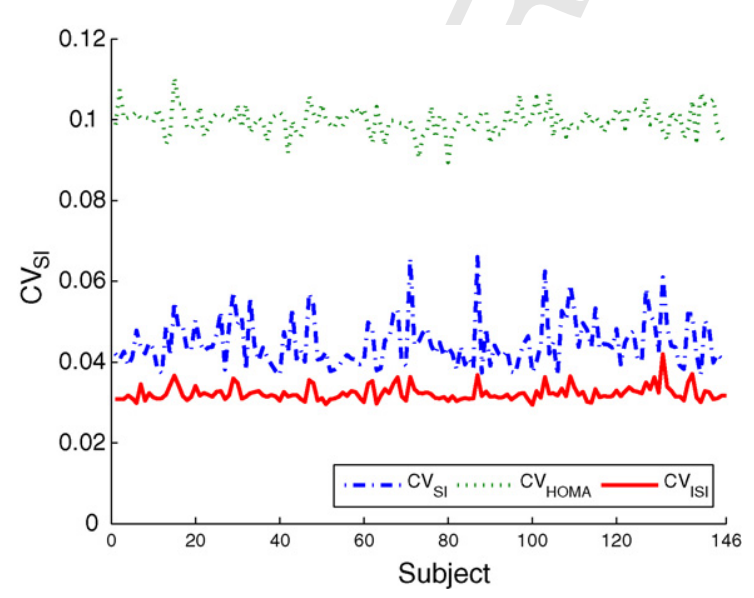

Fig. 5 - Intra-individual coefficients of variation for $S_{I-M C}$ $\left(\mathrm{CV}_{\mathrm{SI}}\right), \mathrm{ISI}_{\mathrm{G}}\left(\mathrm{CV}_{\mathrm{ISI}}\right)$ and HOMA (CV $\left.\mathrm{CV}_{\text {HOMA }}\right)$.

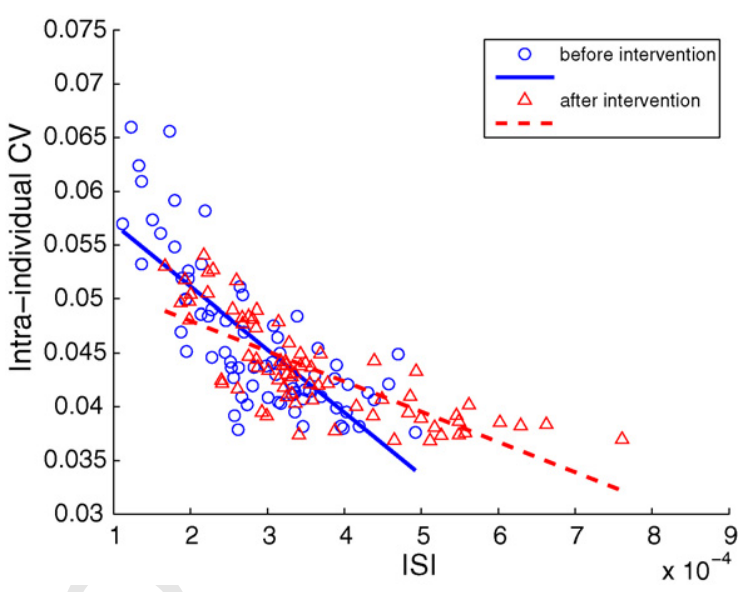

Fig. 6 - Relationship between ISI $_{G}$ and intra-individual coefficients of variation $\mathrm{CV}_{\mathrm{SI}}$ before and after intervention.

The increase in insulin sensitivity after lifestyle intervention [24] was captured by the model, with $S_{\text {I }}$ increasing from $\mathrm{S}_{\mathrm{I}-\mathrm{MC}(\mathrm{BEFORE})}=4.99$ (S.D. 1.47$) \times 10^{-4} \mathrm{lmU}^{-1} \mathrm{~min}^{-1}$ to $\mathrm{S}_{\text {I-MC(AFTER) }}=6.19$ (S.D. 2.02) $\times 10^{-4} \mathrm{lmU}^{-1} \mathrm{~min}^{-1}$. This value matches the increase in ISI shown in Table 1 . Correlation between the change in $\mathrm{S}_{\mathrm{I}-\mathrm{MC}}$ and $\mathrm{ISI}_{\mathrm{G}}, \Delta \mathrm{S}_{\mathrm{I}-\mathrm{MC}}$ and $\Delta \mathrm{ISI}_{\mathrm{G}}$ is $r=0.96$ (90\% CI: 0.96-0.97) with a mean regression line of

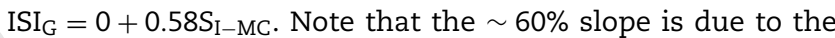
fixed $3 / 5$ ratio of insulin concentration in interstitium $(Q)$ and plasma (I) during steady state. Specifically, ISI is calculated using $I$ and $S_{I}$ is identified using $Q$.

The intra-individual CV of $\mathrm{S}_{\mathrm{I}-\mathrm{MC}}$ decreased slightly from $\mathrm{CV}_{\mathrm{SI}(\mathrm{BEFORE})}=4.6 \% \quad(90 \%$ CI: $3.8-5.9 \%)$ to $\mathrm{CV}_{\mathrm{SI}(\mathrm{AFTER})}=4.3 \%$ (90\% CI: $3.7-5.2 \%)$. A strong correlation of $r=0.83$ could be seen between a decrease in insulin sensitivity ISI and intraindividual CV in $\mathrm{S}_{\mathrm{I}-\mathrm{MC}}$. Fig. 6 shows the linear relationships between ISI $_{G}$ and $\mathrm{CV}_{\mathrm{SI}}$ before and after intervention. A clear reduction in accuracy of estimated $S_{I}$ can be seen in subjects with very low insulin sensitivities.

Re-simulating the low intensity test protocol with different doses of glucose and insulin showed a clear dependence of accuracy of the method on the dose employed, as can be seen in Fig. 7. Administering $5 \mathrm{~g}$ glucose and $0.5 \mathrm{U}$ insulin resulted in $\mathrm{CV}_{\mathrm{SI}}=6.9 \%$ (90\% CI: 4.9-9.9\%). The high dose variant with $20 \mathrm{~g}$ glucose and $2 \mathrm{U}$ insulin resulted in a more accurate measure with $\mathrm{CV}_{\mathrm{SI}}=3.6 \%(90 \% \mathrm{CI}: 3.0-4.5 \%)$, which is very close to the accuracy of ISI. Correlation of $\mathrm{CV}_{\mathrm{SI}}$ with $\mathrm{ISI}_{\mathrm{G}}$ was stronger in the low dose protocol $(r=0.90)$ but showed a weaker linear relationship in the high dose variant $(r=0.46)$.

Simulated hepatic insulin clearance $n_{\mathrm{L}}$ and simulated first pass hepatic insulin extraction $x$ were underestimated slightly in the Monte Carlo analysis, by $-4.4 \%$ (90\% CI: $-16.5-8.1 \%)$ and $-2.2 \%$ (90\% CI: $-12.4-7.3 \%)$, respectively. Simulated glucose distribution volume $\mathrm{V}_{\mathrm{G}}$ was overestimated by $1.7 \%(90 \%$ CI: $0.7-3.5 \%)$.

\section{Discussion}

The model-based protocol presented was developed with the main goal to provide a clinically useful, highly accurate 

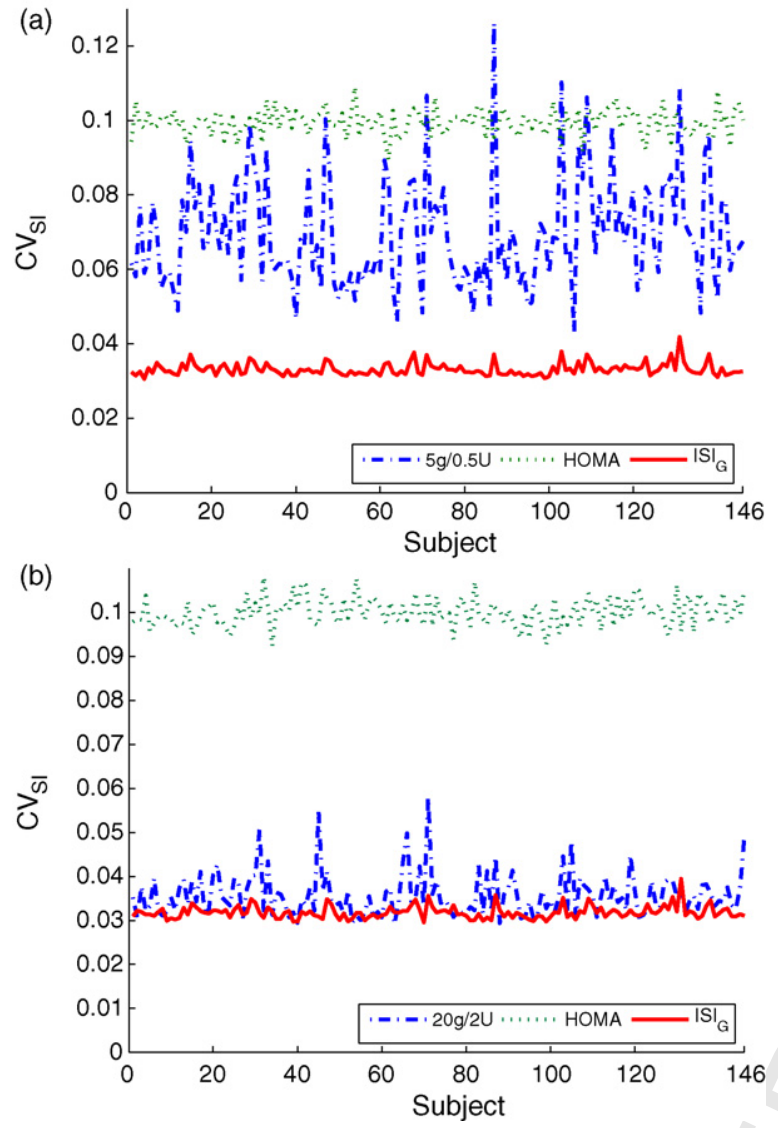

Fig. $7-\mathrm{CV}_{\text {SI }}$ compared to $\mathrm{CV}_{\text {ISI }}$ and $\mathrm{CV}_{\text {HOMA }}$ simulating the protocol with $5 \mathrm{~g}$ glucose and $0.5 \mathrm{U}$ insulin (above), and $20 \mathrm{~g}$ glucose and $2 \mathrm{U}$ insulin (below).

method to diagnose insulin resistance (IR), that is highly correlated to the gold-standard euglycaemic-hyperinsulinaemic clamp. To be clinically useful, a test must be accurate, short and simple. To correlate highly to the clamp, a test must measure the same effects as the clamp. The most widely used and accepted tests developed so far (e.g. IVGTT, OGTT and HOMA), are all judged by their ability to correlate to the clamp. This goal has been achieved only with some significant variability, yielding a wide range of IVGTT-clamp correlations between $r=0.44-0.89$ (e.g. [62-64]).

A main obstacle is that every test effectively measures a different effect [4]. The clamp relies on a steady state glucose concentration during supra-physiological insulin and glucose infusions, in which endogenous insulin and glucose are assumed to be completely suppressed. Its metric for insulin sensitivity is the rate at which glucose is disposed in the body with a given plasma insulin concentration. In contrast, the IVGTT fits the Minimal Model [6] to the glucose response curve after a high-dose injection of glucose and insulin by estimating three model parameters $\left(p_{1}, p_{2}, p_{3}\right)$, with insulin sensitivity being the ratio $S_{I-M M}=p_{3} / p_{2}$. Fitting three parameters has the disadvantage that a longer test is required to allow for enough resolution and data and thus inter-subject variability is distributed amongst these three parameters. In general, the IVGTT is considered the best clamp-correlated method, with correlation values of up to $r=0.89$ being reported [64]. However, lower results as low as $r=0.44$ have also been reported [62].

Other popular methods, widely used due to their simplicity, are surrogate measures such as the OGTT (measuring the rate of glucose decay after an oral glucose load) and HOMA (based on one fasting glucose and insulin sample). These methods are less correlated to the clamp, as they too measure different effects. In particular, HOMA can be very variable due to a pulsatile secretion of insulin [16] and assay inaccuracies, leading to a $\mathrm{CV}>10 \%$ [17].

The proposed low intensity protocol presented was designed to specifically measure the same effects as the clamp in a much shorter and less intense transient test. Variability is constrained to insulin dependent effects in the periphery, controlled by the insulin sensitivity parameter $\mathrm{S}_{\mathrm{I}}$. Modeled $\mathrm{S}_{\mathrm{I}}$ is lower than clamp ISI, but it does not introduce additional variability. The difference is consistent across all individuals, due to the fixed ratio of steady state plasma (I) and interstitial $(\mathrm{Q})$ insulin in the model. The model and fitting method employed have been well validated $[26,22]$ and correlated to clamp data in transient and steady state [31], resulting in very high correlations $(r=0.97$ in transient state, $r=0.99$ in steady state) [31].

The proposed method was able to estimate $S_{I}$ with high accuracy, given the assay errors and unmodeled suppression of EGP. $\mathrm{CV}_{\mathrm{SI}}$ was slightly larger than $\mathrm{CV}_{\text {ISI }}$. This larger $\mathrm{CV}$ can be expected given the highly dynamic state of the proposed test. Accuracy decreased drastically by $53 \%$ in the lower dose test ( $5 \mathrm{~g}$ glucose, $0.5 \mathrm{U}$ insulin), though accuracy was still better than HOMA. The higher dose test $(20 \mathrm{~g}$ glucose, $2 \mathrm{U}$ insulin) improved accuracy by $20 \%$. As suppression of EGP was adjusted to the dose accordingly, being higher in the high dose test, the still improved accuracy suggests a strong dependence on the signal to noise ratio of the test, with EGP playing a minor role.

In spite of the improved accuracy at higher dose, it is not as practical for a simple clinical test, for a variety of reasons. As IV glucose is commonly available in $50 \%$ solution, $20 \mathrm{~g}$ requires a $40 \mathrm{ml}$ injection of a very viscous solution, which causes discomfort for the test subject. The $2 \mathrm{U}$ insulin dose also increases risk of hypoglycaemia, particularly in lean subjects. Finally, an intravenous glucose bolus of $20 \mathrm{~g}$ is on the upper physiological range, possibly triggering other glucose regulatory effects not accounted for in this simulation, which could in reality worsen results. The $10 \mathrm{~g}$ glucose and $1 \mathrm{U}$ insulin dose is only slightly less accurate, but a lot easier and safer to administer in clinical practice. Mean and range of $\mathrm{CV}_{\mathrm{SI}}$ are greatly reduced in the step from low to medium dose, whereas the improvement from medium to high dose is not as pronounced any more. This decay is shown in Fig. 8, which illustrates that the medium dose of $10 \mathrm{~g}$ glucose and $1 \mathrm{U}$ insulin appears to be the best compromise in practicability, safety and accuracy.

A strong negative correlation was seen between a decrease in insulin sensitivity $\mathrm{ISI}_{\mathrm{G}}$ and $\mathrm{CV}_{\mathrm{SI}}$. This correlation was even stronger with the low dose test, but was markedly reduced in the high dose test. The origin of this effect is likely physiological, as insulin-dependent effects are less dominant in subjects with low insulin sensitivity, leading to a reduced sig- 


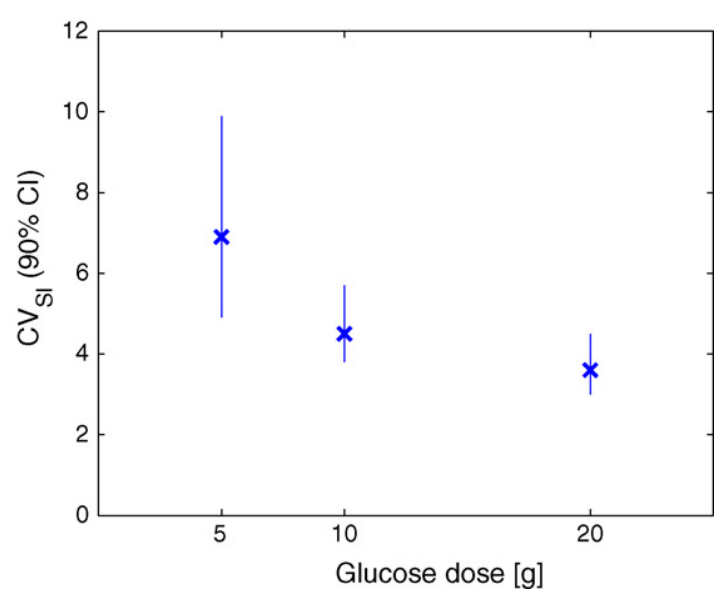

Fig. $8-\mathrm{CV}_{\mathrm{SI}}$ with the $90 \%$ confidence intervals for the low (5 g glucose, $0.5 \mathrm{U}$ insulin), medium (10 g glucose, $1 \mathrm{U}$ insulin) and high ( $20 \mathrm{~g}$ glucose, $2 \mathrm{U}$ insulin) dose test variants.

nal to noise ratio. As can be seen with the high dose test, this correlation can be reduced by increasing the signal. In contrast, the correlation is stronger with a weaker signal as shown in the low dose test.

Overall, the method is able to estimate the underlying insulin sensitivity with high accuracy from the proposed test protocol. Results from the first clinical pilot trials [23] are shown in Fig. 9 and suggest an equally good performance in fitting the dose-response data from real clinical data. In the example shown, the proposed method estimated virtually the same $S_{I}$ in two tests on the same subject. The two tests were separated by a 2 week interval. Whether the estimated $S_{I}$ is a true marker of insulin sensitivity will require further clin- ical validation with the clamp and other methods. However, judging from the accurate simulation of clinically observed dynamics, it is very likely that the effect described by the model parameter $S_{I}$ is physiological and that insulin sensitivity can be estimated with similar accuracy in clinical data. This result is supported by the high correlation between $S_{I}$ and ISI using clamp test data [31]. More specifically, because the proposed low intensity test was specifically designed to measure the same physiological effect as the clamp using highly correlated models and methods, the test should also be highly correlated to the clamp.

Even if the most prominent unmodeled dynamic (suppression of EGP) is included in this Monte Carlo analysis, real results could still be affected by other effects not simulated here. Inaccuracies in the simulated test protocol were identified in initial trials [23], i.e. in sample timing and imperfect cannula flushing, or incomplete mixing of glucose and insulin in plasma during the first $10 \mathrm{~min}$. These effects are more likely in a clinical, non-research setting with a simple proto$\mathrm{col}$, where special considerations common in research settings cannot be met. These factors have to be taken into account when designing a robust clinical test. Additional variability could be introduced by less accurate assay methods, especially for insulin and C-peptide. The assays used in this study are run by the authors' collaborating laboratory and are amongst the most accurate methods. Less accurate insulin assays with more cross reactivity to proinsulin are still widely used and could increase the test's variability or introduce a systematic error $[65,66]$.

Finally, in a simulation setting means are limited and not all noise and physiological dynamics can be accounted for. We have tried to best possibly approximate a real clinical setting, but ongoing pilot studies and a complete clinical validation against the gold-standard clamp test will have to be completed to fully validate these simulation results.
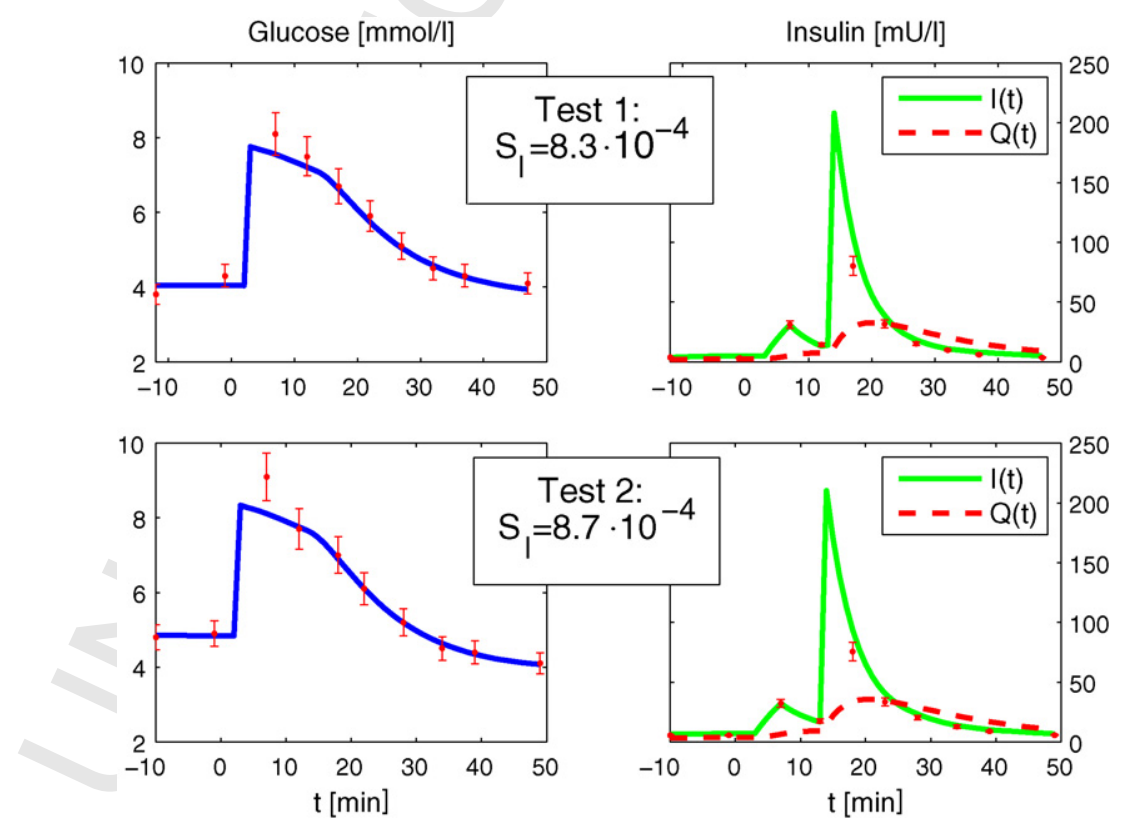

Fig. 9 - Example of two pilot clinical tests of the proposed protocol on one subject. Test 1 is shown above and test 2 below. The left side shows plasma glucose concentrations and the right side plasma insulin concentrations (measurements and model fitted profiles). Modeled insulin profiles shown are in plasma (I(t), solid) and in interstitial fluid ( $Q(t)$, dashed). 


\section{Conclusions}

The proposed method to diagnose insulin resistance proved to be very accurate in Monte Carlo simulation, and only slightly less accurate than the gold-standard clamp test. As a result of its design to measure the same effects as the clamp, it is highly correlated to the gold-standard clamp ISI metric. The physiological dosing, simple and robust protocol and high accuracy make it very attractive for early diagnosis and monitoring of interventions. Accuracy and correlation to gold-standard tests in a clinical setting must still be assessed. However, this study has indicated that the proposed test should possess the accuracy and robustness required, as compared to a large cohort of clamp results.

\section{REFERENCES}

[1] R.A. DeFronzo, E. Ferrannini, Insulin resistance. A multifaceted syndrome responsible for NIDDM, obesity, hypertension, dyslipidemia, and atherosclerotic cardiovascular disease, Diabetes Care 14 (3) (1991) 173-194.

[2] R.A. DeFronzo, J.D. Tobin, R. Andres, Glucose clamp technique: a method for quantifying insulin secretion and resistance, Am. J. Physiol. 237 (3) (1979) E214-E223.

[3] E. Ferrannini, A. Mari, How to measure insulin sensitivity, J. Hypertens. 16 (7) (1998) 895-906.

[4] J. Radziuk, Insulin sensitivity and its measurement: structural commonalities among the methods, J. Clin. Endocrinol. Metab. 85 (12) (2000) 4426-4433.

[5] E. Bonora, P. Moghetti, C. Zancanaro, M. Cigolini, M. Querena, V. Cacciatori, A. Corgnati, M. Muggeo, Estimates of in vivo insulin action in man: comparison of insulin tolerance tests with euglycemic and hyperglycemic glucose clamp studies, J. Clin. Endocrinol. Metab. 68 (2) (1989) 374-378.

[6] R.N. Bergman, Y.Z. Ider, C.R. Bowden, C. Cobelli, Quantitative estimation of insulin sensitivity, Am. J. Physiol. 236 (6) (1979) E667-E677.

[7] J.C. Beard, R.N. Bergman, W.K. Ward, D.J. Porte, The insulin sensitivity index in nondiabetic man. Correlation between clamp-derived and IVGTT-derived values, Diabetes 35 (1986) 362-369.

[8] Y.J. Yang, J.H. Youn, R.N. Bergman, Modified protocols improve insulin sensitivity estimation using the minimal model, Am. J. Physiol. 253 (6 Pt 1) (1987) E595-E602.

[9] R.N. Bergman, D.T. Finegood, M. Ader, Assessment of insulin sensitivity in vivo, Endocr. Rev. 6 (1) (1985) 45-86.

[10] M. Matsuda, R.A. DeFronzo, Insulin sensitivity indices obtained from oral glucose tolerance testing: comparison with the euglycemic insulin clamp, Diabetes Care 22 (9) (1999) 1462-1470.

[11] C. Dalla Man, A. Caumo, C. Cobelli, The oral glucose minimal model: Estimation of insulin sensitivity from a meal test, IEEE Trans. Biomed. Eng. 49 (5) (2002) 419-429.

[12] G. Pacini, R.N. Bergman, MINMOD: a computer program to calculate insulin sensitivity and pancreatic responsivity from the frequently sampled intravenous glucose tolerance test, Comput. Methods Programs Biomed. 23 (2) (1986) 113-122.

[13] G. Pacini, A. Mari, Methods for clinical assessment of insulin sensitivity and beta-cell function, Best Pract. Res. Clin. Endocrinol. Metab. 17 (3) (2003) 305-322.

[14] D.R. Matthews, J.P. Hosker, A.S. Rudenski, B.A. Naylor, D.F. Treacher, R.C. Turner, Homeostasis model assessment: insulin resistance and beta-cell function from fasting plasma glucose and insulin concentrations in man, Diabetologia 28 (7) (1985) 412-419.

[15] A. Katz, S.S. Nambi, K. Mather, A.D. Baron, D.A. Follmann, G. Sullivan, M.J. Quon, Quantitative insulin sensitivity check index: a simple, accurate method for assessing insulin sensitivity in humans, J. Clin. Endocrinol. Metab. 85 (7) (2000) 2402-2410.

[16] S.H. Song, S.S. McIntyre, H. Shah, J.D. Veldhuis, P.C. Hayes, P.C. Butler, Direct measurement of pulsatile insulin secretion from the portal vein in human subjects, J. Clin. Endocrinol. Metab. 85 (12) (2000) 4491-4499.

[17] T.M. Wallace, J.C. Levy, D.R. Matthews, Use and abuse of HOMA modeling, Diabetes Care 27 (6) (2004) 1487-1495.

[18] T.M. Wallace, J.C. Levy, D.R. Matthews, An increase in insulin sensitivity and basal beta-cell function in diabetic subjects treated with pioglitazone in a placebo-controlled randomized study, Diab. Med. 21 (6) (2004) 568-576.

[19] E. Ferrannini, A. Mari, Beta cell function and its relation to insulin action in humans: a critical appraisal, Diabetologia 47 (5) (2004) 943-956.

[20] ADA, Diagnosis and classification of diabetes mellitus, Diabetes Care 29 (S1) (2006) S43-8.

[21] E. Ferrannini, Insulin resistance is central to the burden of diabetes, Diabetes Metab. Rev. 13 (2) (1997) 81-86.

[22] T. Lotz, J.G. Chase, J. Lin, X.W. Wong, C.E. Hann, K.A. McAuley, S. Andreassen, Integral-based identification of a physiological insulin and glucose model on euglycaemic clamp trials, in: 14th IFAC Symposium on System Identification (SYSID 2006), IFAC, Newcastle, Australia, 2006, pp. 463-468.

[23] T.F. Lotz, J.G. Chase, K.A. McAuley, G.M. Shaw, C.E. Hann, J.I. Mann, A highly correlated method to assess insulin resistance in broad populations, in: 12th International Conference on Biomedical Engineering (ICBME), Singapore, 2005.

[24] K.A. McAuley, S.M. Williams, J.I. Mann, A. Goulding, A. Chisholm, N. Wilson, G. Story, R.T. McLay, M.J. Harper, I.E. Jones, Intensive lifestyle changes are necessary to improve insulin sensitivity: a randomized controlled trial, Diabetes Care 25 (3) (2002) 445-452.

[25] M. Ader, R.N. Bergman, Insulin sensitivity in the intact organism, Baillieres Clin. Endocrinol. Metab. 1 (4) (1987) 879-910.

[26] C.E. Hann, J.G. Chase, J. Lin, T. Lotz, C.V. Doran, G.M. Shaw, Integral-based parameter identification for long-term dynamic verification of a glucose-insulin system model, Comput. Methods Programs Biomed. 77 (3) (2005) 259-270.

[27] J.G. Chase, G.M. Shaw, J. Lin, C.V. Doran, C. Hann, M.B. Robertson, P.M. Browne, T. Lotz, G.C. Wake, B. Broughton, Adaptive bolus-based targeted glucose regulation of hyperglycaemia in critical care, Med. Eng. Phys. 27 (1) (2005) $1-11$.

[28] X.W. Wong, I. Singh-Levett, L.J. Hollingsworth, G.M. Shaw, C.E. Hann, T. Lotz, J. Lin, O.S. Wong, J.G. Chase, A novel, model-based insulin and nutrition delivery controller for glycemic regulation in critically ill patients, Diabetes Technol. Ther. 8 (2) (2006) 174-190.

[29] T. Lonergan, A. LeCompte, M. Willacy, J.G. Chase, G.M. Shaw, X.W. Wong, T. Lotz, J. Lin, C.E. Hann, A simple insulin-nutrition protocol for tight glycemic control in critical illness: development and protocol comparison, Diabetes Technol. Ther. 8 (2) (2006) 191-206.

[30] T. Lonergan, A.L. Compte, M. Willacy, J.G. Chase, G.M. Shaw, C.E. Hann, T. Lotz, J. Lin, X.W. Wong, A pilot study of the SPRINT protocol for tight glycemic control in critically Ill patients, Diabetes Technol. Ther. 8 (4) (2006) 449-462.

[31] T.F. Lotz, J.G. Chase, K.A. McAuley, D.S. Lee, J. Lin, C.E. Hann, J.I. Mann, Transient and steady-state euglycemic clamp 
validation of a model for glycemic control and insulin sensitivity testing, Diabetes Technol. Ther. 8 (3) (2006) 338-346.

[32] R.L. Prigeon, M.E. Roder, J. Porte, D.S.E. Kahn, The effect of insulin dose on the measurement of insulin sensitivity by the minimal model technique. Evidence for saturable insulin transport in humans, J. Clin. Invest. 97 (2) (1996) 501-507.

[33] A. Natali, A. Gastaldelli, S. Camastra, A.M. Sironi, E. Toschi, A. Masoni, E. Ferrannini, A. Mari, Dose-response characteristics of insulin action on glucose metabolism: a non-steady-state approach, Am. J. Physiol. Endocrinol. Metab. 278 (5) (2000) E794-E801.

[34] R.S. Sherwin, K.J. Kramer, J.D. Tobin, P.A. Insel, J.E. Liljenquist, M. Berman, R. Andres, A model of the kinetics of insulin in man, J. Clin. Invest. 53 (5) (1974) 1481-1492.

[35] A.C. Guyton, J.E. Hall, Textbook of Medical Physiology, 9th ed., W.B. Saunders Company, 1996.

[36] E. Van Cauter, F. Mestrez, J. Sturis, K.S. Polonsky, Estimation of insulin secretion rates from C-peptide levels. Comparison of individual and standard kinetic parameters for C-peptide clearance, Diabetes 41 (3) (1992) 368-377.

[37] S. Gudbjornsdottir, M. Sjostrand, L. Strindberg, J. Wahren, P. Lonnroth, Direct measurements of the permeability surface area for insulin and glucose in human skeletal muscle, J. Clin. Endocrinol. Metab. 88 (10) (2003) 4559-4564.

[38] M. Sjostrand, A. Holmang, P. Lonnroth, Measurement of interstitial insulin in human muscle, Am. J. Physiol. 276 (1 Pt 1) (1999) E151-E154.

[39] M. Sjostrand, S. Gudbjornsdottir, L. Strindberg, P. Lonnroth, Delayed transcapillary delivery of insulin to muscle interstitial fluid after oral glucose load in obese subjects, Diabetes 54 (1) (2005) 152-157.

[40] C.V. Doran, Modelling and control of hyperglycemia in critical care patients, Masters of Engineering (ME), University of Canterbury, 2004.

[41] J.G. Chase, G.M. Shaw, J. Lin, C.V. Doran, C. Hann, T. Lotz, G.C. Wake, B. Broughton, Targeted glycemic reduction in critical care using closed-loop control, Diabetes Technol. Ther. 7 (2) (2005) 274-282.

[42] X.W. Wong, J.G. Chase, G.M. Shaw, C.E. Hann, T. Lotz, J. Lin, I. Singh-Levett, L.J. Hollingsworth, O.S. Wong, S. Andreassen, Model predictive glycaemic regulation in critical illness using insulin and nutrition input: a pilot study, Med. Eng. Phys. 28 (7) (2006) 665-681.

[43] C. Hann, J. Chase, G. Shaw, Integral-based Identification of Patient Specific Parameters for a Minimal Cardiac Model, Comput. Methods Programs Biomed. 81 (2) (2006) 181-192.

[44] B. Thorsteinsson, Kinetic models for insulin disappearance from plasma in man, Dan Med. Bull. 37 (2) (1990) 143-153.

[45] M.E. Valera Mora, A. Scarfone, M. Calvani, A.V. Greco, G. Mingrone, Insulin clearance in obesity, J. Am. Coll. Nutr. 22 (6) (2003) 487-493.

[46] C. Waterhouse, J. Keilson, Transfer times across the human body, Bull. Math Biophys. 34 (1) (1972) 33-44.

[47] A. Despopoulos, S. Silbernagl, Color atlas of physiology, 5th ed., Thieme Flexibook, G. Thieme Thieme Medical Publishers, Stuttgart, New York, 2003.

[48] G.M. Argoud, D.S. Schade, R.P. Eaton, Insulin suppresses its own secretion in vivo, Diabetes 36 (8) (1987) 959-962.

[49] L.S. Jefferson, A. Cherrington, The endocrine pancreas and regulation of metabolism, Handbook of Physiology-The Endocrine System, vol. 2, Oxford University Press, Oxford, 2001.

[50] R.P. Eaton, R.C. Allen, D.S. Schade, K.M. Erickson, J. Standefer, Prehepatic insulin production in man: kinetic analysis using peripheral connecting peptide behavior, J. Clin. Endocrinol. Metab. 51 (3) (1980) 520-528.

[51] A. Mari, Assessment of insulin sensitivity and secretion with the labelled intravenous glucose tolerance test: improved modelling analysis, Diabetologia 41 (9) (1998) 1029-1039.

[52] E.R. Carson, C. Cobelli, Modelling methodology for physiology and medicine, Academic Press Series in Biomedical Engineering, Academic Press, San Diego, 2001.

[53] E. Ferrannini, C. Cobelli, The kinetics of insulin in man. II. Role of the liver, Diabetes Metab. Rev. 3 (2) (1987) 365-397.

[54] J.J. Meier, J.D. Veldhuis, P.C. Butler, Pulsatile insulin secretion dictates systemic insulin delivery by regulating hepatic insulin extraction in humans, Diabetes 54 (6) (2005) 1649-1656.

[55] G. Toffolo, M. Campioni, R. Basu, R.A. Rizza, C. Cobelli, A minimal model of insulin secretion and kinetics to assess hepatic insulin extraction, Am. J. Physiol. Endocrinol. Metab. 290 (1) (2006) E169-E176.

[56] L.A. Trissel, Drug compatibility differences with propofol injectable emulsion products, Crit. Care Med. 29 (2) (2001) 466-468.

[57] A. Vella, A.S. Reed, N. Charkoudian, P. Shah, R. Basu, A. Basu, M.J. Joyner, R.A. Rizza, Glucose-induced suppression of endogenous glucose production: dynamic response to differing glucose profiles, Am. J. Physiol. Endocrinol. Metab. 285 (1) (2003) E25-E30.

[58] P. Vicini, J.J. Zachwieja, K.E. Yarasheski, D.M. Bier, A. Caumo, C. Cobelli, Glucose production during an IVGTT by deconvolution: validation with the tracer-to-tracee clamp technique, Am. J. Physiol. 276 (2 Pt 1) (1999) E285-E294.

[59] S. Nagasaka, K. Tokuyama, I. Kusaka, H. Hayashi, K. Rokkaku, T. Nakamura, A. Kawakami, M. Higashiyama, S. Ishikawa, T. Saito, Endogenous glucose production and glucose effectiveness in type 2 diabetic subjects derived from stable-labeled minimal model approach, Diabetes 48 (5) (1999) 1054-1060.

[60] Roche, Data Sheet-Insulin Immunoassay, Elecsys 1010/2010/Modular Analytics E170, Tech. Rep. 12017547 122, Roche Diagnostics, Mannheim, Germany (2004).

[61] Roche, Data Sheet-C-Peptide Immunoassay, Elecsys 1010/2010/Modular Analytics E170, Tech. Rep. 03184897 190, Roche Diagnostics, Mannheim, Germany (2005).

[62] C.C. Donner, E. Fraze, Y.D. Chen, C.B. Hollenbeck, J.E. Foley, G.M. Reaven, Presentation of a new method for specific measurement of in vivo insulin-stimulated glucose disposal in humans: comparison of this approach with the insulin clamp and minimal model techniques, J. Clin. Endocrinol. Metab. 60 (4) (1985) 723-726.

[63] M.F. Saad, R.L. Anderson, A. Laws, R.M. Watanabe, W.W. Kades, I.G.Y.-D $D_{-\bar{k}}$ R.E. Sands, D. Pei, P.J. Savage, R.N. Bergman, A comparison between the minimal model and the glucose clamp in the assessment of insulin sensitivity across the spectrum of glucose tolerance, Diabetes 43 (1994) 1114-21.

[64] R.N. Bergman, R. Prager, A. Volund, J.M. Olefsky, Equivalence of the insulin sensitivity index in man derived by the minimal model method and the euglycemic glucose clamp, J. Clin. Invest. 79 (3) (1987) 790-800.

[65] D.C. Robbins, L. Andersen, R. Bowsher, R. Chance, B. Dinesen, B. Frank, R. Gingerich, D. Goldstein, H.M. Widemeyer, S. Haffner, C.N. Hales, L. Jarett, K. Polonsky, D. Porte, J. Skyler, G. Webb, K. Gallagher, Report of the American Diabetes Association's Task Force on standardization of the insulin assay, Diabetes 45 (2) (1996) 242-256.

[66] D. Chevenne, F. Trivin, D. Porquet, Insulin assays and reference values, Diabetes Metab. 25 (6) (1999) 459-476. 